\title{
ERRATUM
}

To the paper:

\section{Time to call for a global public health approach in prevention of the onset and progression of problematic gaming}

THOMAS CHUNG, SIMMY SUM and MONIQUE CHAN

published in the Journal of Behavioral Addictions, 7(3), pp. 548-552 (2018), DOI: 10.1556/2006.7.2018.86

The city name of the affiliation has been published improperly.

The correct affiliation is as follows:

THOMAS CHUNG*, SIMMY SUM and MONIQUE CHAN

Student Health Service, Department of Health, Hong Kong SAR, China 